\title{
Early Cognitive Dysfunction in Elderly Patients after Total Knee Arthroplasty: An Analysis of Risk Factors and Cognitive Functional Levels
}

\author{
Shengjie Ren, ${ }^{1}$ Fang Yuan, ${ }^{1}$ Shu Yuan, ${ }^{1}$ Chuanbo Zang, ${ }^{1}$ Yanan $Z$ hang, ${ }^{1}$ and Bao Lang $\mathbb{D}^{2}$ \\ ${ }^{1}$ School of Anesthesiology, Weifang Medical University, Weifang 261053, China \\ ${ }^{2}$ Department of Anesthesiology, Weifang People's Hospital, Weifang 261041, China \\ Correspondence should be addressed to Bao Lang; wfrmyy91@163.com
}

Received 26 August 2021; Revised 11 December 2021; Accepted 28 January 2022; Published 18 February 2022

Academic Editor: Cheng $\mathrm{Ni}$

Copyright ( 2022 Shengjie Ren et al. This is an open access article distributed under the Creative Commons Attribution License, which permits unrestricted use, distribution, and reproduction in any medium, provided the original work is properly cited.

\begin{abstract}
Background. Cognitive dysfunction after total knee arthroplasty (TKA) is very common in elderly patients. Postoperative cognitive dysfunction (POCD), as a form of cognitive dysfunction, may affect patients' short- and long-term recoveries. The identification of meaningful risk factors may help reduce the occurrence of POCD in the future. Objective. Our goal was to retrospectively investigate the risk factors for early POCD in elderly patients undergoing TKA and to further analyze the relationship between the intensity of risk factors and the level of cognitive function. Methods. The related indicators and the Montreal Cognitive Function Assessment Scale (MOCA) scores of 105 elderly patients were collected by searching the electronic case system. According to the postoperative MOCA score, patients were divided into three groups: normal group (group N), mild POCD group (group M), and severe POCD group (group S). SPSS 25.0 software was used for statistical analyses. Results. At baseline, the preoperative MOCA score was significantly different in patients with POCD $(P \leq 0.001)$, while other baseline indicators were not significantly different. In terms of changes in hemoglobin levels, statistically significant differences were observed between group $\mathrm{M}$, group $\mathrm{S}$, and group $\mathrm{N}(P=0.039)$. Among inflammatory indicators, only postoperative CRP levels showed a statistically significant difference in patients with POCD $(P=0.041)$. Postoperative pain was also significantly different among the three groups $(P=0.009)$. The multivariate regression analysis revealed that a low preoperative MOCA score and severe postoperative pain were independent risk factors for mild and severe cognitive impairment, while a high postoperative CRP level was only an independent risk factor for mild cognitive impairment. Conclusions. Our study found that the level of preoperative cognitive function, postoperative CRP level, and postoperative pain were independent risk factors for POCD. Moreover, the levels of preoperative cognitive function and postoperative pain were more strongly correlated with severe POCD than postoperative CRP levels.
\end{abstract}

\section{Introduction}

With the aging of the population and the improvement of people's living standards, total knee arthroplasty (TKA) is being increasingly widely implemented in elderly patients. It successfully improves quality of life by reducing pain and improving long-term function. However, as with all surgeries, TKA may result in complications, including thromboembolism, bone-cement syndrome, anemia, cardiac arrhythmia, pain, and POCD.
POCD exerts an effect on a wide range of cognitive domains, such as memory, attention, orientation, and concentration, but a uniform definition of POCD seems to be lacking and the length of follow-up period limits comparability between studies, which also increase the challenges associated with research in this field [1]. A large number of studies have concluded that the incidence of POCD ranges from $10 \%$ to $54.3 \%$ [2]. The wild range is also related to the inconclusive diagnosis of POCD. To address this issue, the Montreal cognitive assessment (MOCA), with its high 
sensitivity and specificity, was developed as a tool to screen patients with mild cognitive impairment and whose MMSE is generally within the normal range [3].

POCD markedly impairs postoperative recovery, increases mortality, and increases the utilization of social and financial resources [4]. The specific mechanism of POCD remains unclear, but its related risk factors have been continuously explored. According to recent studies, in terms of the individual characteristics, age, educational level, mental health, and comorbidities may be the risk factors for POCD. In terms of surgery, hypotension, procedure time, blood loss, and a low temperature may increase the POCD incidence. In terms of anesthesia, methods of anesthesia and anesthetic drugs also affect the postoperative cognitive function. Although many studies have focused on determining the risk factors for POCD, a comprehensive analysis of risk factors in certain surgeries seems to be more useful in clinical practice. Therefore, the purpose of this study is to investigate the incidence and risk factors for early POCD and even severe POCD in elderly patients undergoing TKA.

\section{Methods}

2.1. Setting and Patients. This study protocol was approved by the Medical Institutional Ethics Committee of Weifang People's Hospital and was registered in the China Clinical Trial Center (ChiCTR2100049338). Patients with knee osteoarthritis (aged $\geq 60$ years) who were scheduled to undergo TKA in general anesthesia at the Weifang People's Hospital from April 2021 to May 2021 were retrospectively included in this study. Inclusion criteria were an age greater than 60 years, patients who underwent TKA surgery, had American Society of Anesthesiologists (ASA) grades I and III, and willing and able to complete study questionnaires. Those patients who were unable to complete preoperative and postoperative MOCA evaluations and were clinically diagnosed with a neurological disorder or psychosis were excluded.

\subsection{Anesthesia and Postoperative Treatment. All included} patients were prepared for routing general anesthesia by inserting an intravenous line, and electrocardiogram, noninvasive blood pressure, pulse oximetry, and $\mathrm{P}_{\mathrm{ET}} \mathrm{CO}_{2}$ were monitored. After entering the operating room, $30 \mathrm{ml}$ of $0.3 \%$ ropivacaine was used for ultrasound-guided femoral nerve block, and all patients were administered $30 \mu \mathrm{g}$ of dexmedetomidine as a premedication. Induction anesthesia was achieved with propofol (closed loop target controlled infusion under bispectral index (BIS) monitoring, initial target concentration: $3 \mu \mathrm{g} / \mathrm{ml})$, remifentanil $(1.5 \mu \mathrm{g} / \mathrm{kg}$ ), sufentanil $(10 \mu \mathrm{g})$, and rocuronium $(30 \mathrm{mg})$. The laryngeal mask was inserted when the patient was unconscious and BIS was stable at 40-60. The lungs were ventilated with $70 \%$ oxygen and controlled with a tidal volume of $6-8 \mathrm{ml} / \mathrm{kg}$, and the ventilatory rate was $12-14$ beats per minute. Respiratory parameters were adjusted to maintain a $\mathrm{PaCO}_{2}$ value of 35 to $40 \mathrm{mmHg}$. Propofol (closed loop target controlled infusion under BIS monitoring (40-60)) and remifentanil $(0.1 \mu \mathrm{g} /$ (kg.min))was used to maintain anesthesia. When the joint cavity was opened, controlled hypotension, which was achieved by increasing the depth of anesthesia or antihypertensive drugs, was performed to reduce bleeding, and then, we maintain the target blood pressure until the end of the operation. We monitored brain oxygen saturation to avoid hypoxic ischemic injury in the brain due to hypoperfusion during anesthesia maintenance. We also used NSAIDs for pain therapy and tropisetron to reduce postoperative nausea and vomiting. Subjects were transferred to the postanesthesia care unit (PACU) after extubation. Patient-controlled intravenous analgesic (PCIA) was used for postoperative analgesia. An analgesic pump contained $170 \mu \mathrm{g}$ of dexmedetomidine and $90 \mu \mathrm{g}$ of sufentanil mixed into $100 \mathrm{ml}$ of liquid, pumped at a rate of $2 \mathrm{ml} / \mathrm{h}$. Ten milligrams of imrecoxib was orally administered twice a day in the ward.

2.3. Data Collection. In this study, we divided it into four time points: preoperative (T0), intraoperative (T1), and postoperative days 1 (T2) and 3 (T3). The following data were collected and recorded: (i) demographics: including sex, age, body mass index (BMI), American Society of Anesthesiologists (ASA) physical status and comorbidities (including arrhythmia, hypertension, diabetes, coronary atherosclerotic heart disease (CHD), history of stroke, and digestive system disease) and (ii) clinicopathological parameters: MOCA score was obtained prior to the operation (T0) and postoperative days 1 (T2) and 3(T3). Blood pressure was recorded as systolic blood pressure (SBP) and diastolic blood pressure (DBP), which were obtained at T0, representing the baseline level, and intraoperatively after controlling hypotension (T1). The intraoperative lowest systolic blood pressure (L-SBP) was also a cause for concern. We also calculated the magnitude of reduction in systolic blood pressure (M-SBP) and diastolic blood pressure (M-DBP) using the following specific equation: (preoperative blood pressure - controlled blood pressure)/preoperative blood pressure. Hemoglobin, hemameba, and neutrophil levels were determined at T0, T2, and T3, while C-reactive protein (CRP) levels and the erythrocyte sedimentation rate (ESR) were only determined at T0 and T3. (iii) Surgery data included the surgery duration, second surgery (Yes or No), and pattern of tourniquet use. Tourniquets can be used in two ways; one is the whole tourniquet (long-term) and the other is the tourniquet when hitting the cement (shortterm). (iv) Postoperative follow-up included the degree of pain at rest (according to numeric rating scales (NRS), 1-3 was classified as mild pain, 4-6 as moderate, and 7-10 as severe) at $\mathrm{T} 2$ and $\mathrm{T} 3$.

2.4. Cognitive Functional Assessment and Criteria for POCD. The cognitive functional assessment was conducted at T0, T2, and T3 using MOCA by a trained professional. The MOCA assessment includes 16 items and 11 categories and assesses short-term memory, visuospatial abilities, executive functions, phonemic fluency, verbal abstraction, attention, concentration, working memory, language, and orientation to time and place (Figure 1). Consistent with numerous studies $[5,6]$, a correction for education effects was applied in present study: $\leq 6$ years of education received a total score 


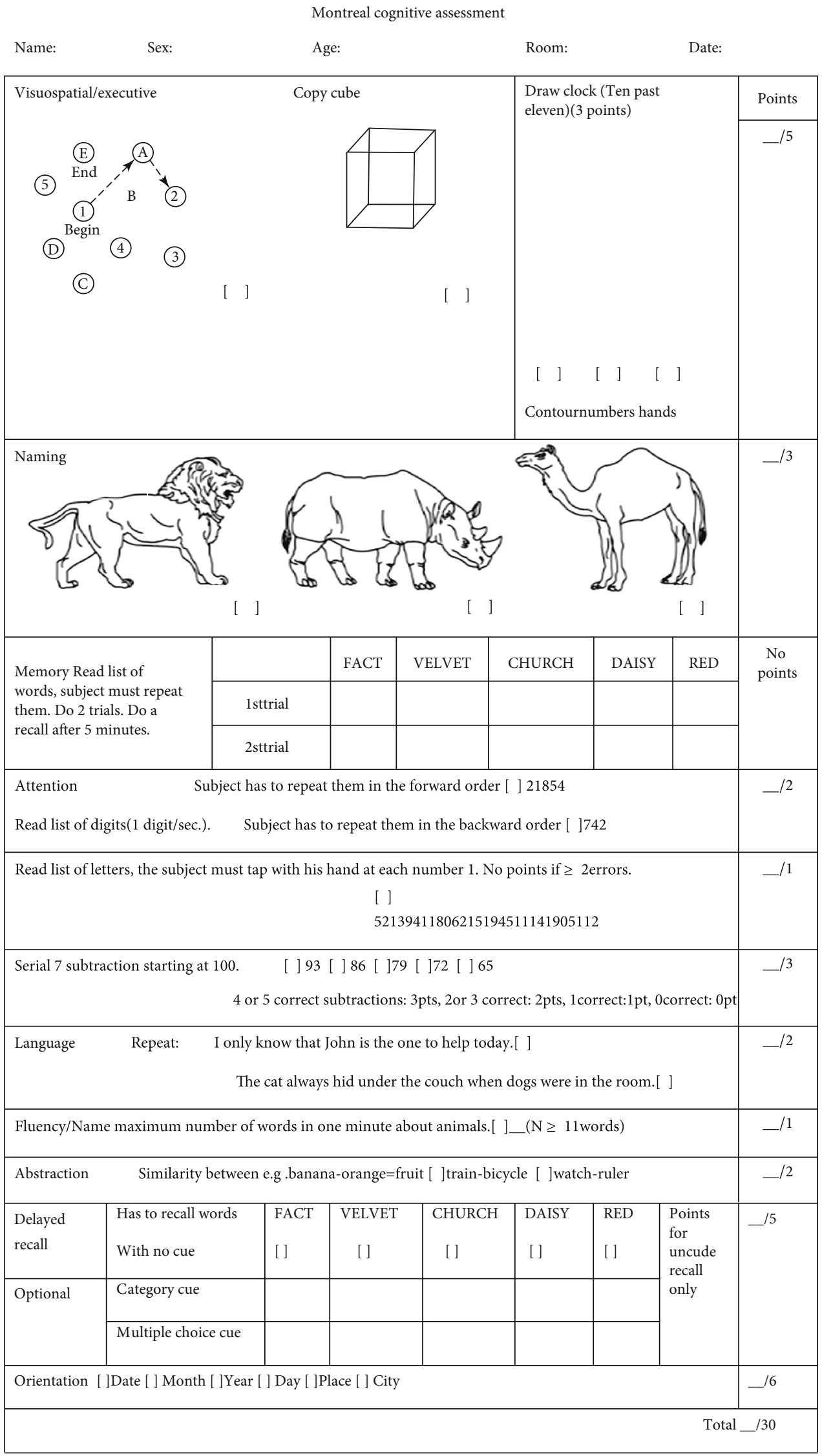

Figure 1: Montreal cognitive assessment (MoCA), Beijing version (in English). 
of +2 and 6 to 12 years of education received a total score of +1 . Higher scores indicate better cognitive function. POCD was defined as a postoperative MOCA score $<26$, as previously described [6-8]. Meanwhile, severe POCD was defined as a MOCA score $<20[9,10]$. Therefore, patients were categorized into a normal group (MOCA scores $\geq 26$, group $\mathrm{N}$ ), a mild POCD group $(20 \leq$ MOCA scores $<26$, group $M)$, and a severe POCD group (MOCA scores $<20$, group $S$ ).

2.5. Statistical Analysis. All data reported in this study were analyzed using SPSS 25.0 software (developed by IBM Corp). For continuous data, normally distributed data are presented as means \pm standard deviations, whereas nonnormally distributed data are presented as medians (interquartile ranges [IQR]). All continuous data were analyzed using the Kruskal-Wallis test. Categorical data are presented as frequencies and proportions and were analyzed using the $\chi^{2}$ test or Fisher-Freeman-Halton test, as appropriate. Three pairwise comparisons were conducted using the KruskalWallis test and univariate ANOVA. Relevant indicators with statistically significant differences in the previous comparison were analyzed using multifactor logistic regression. $P$ $<0.05$ was considered as the statistically significant level.

\section{Result}

3.1. General Information. One hundred five patients were able to participate in the study, and 103 patients were eventually enrolled. The remaining 2 patients were excluded because they did not have complete data. According to postoperative MOCA scores, the 103 patients were divided into three groups, group N (30 cases), group M (40 cases), and group S (33 cases). With the exception of the preoperative MOCA score, no significant differences in the baseline characteristic data were observed between the three groups, such as sex, age, BMI, SBP, DBP, hemoglobin level, ESR, CRP level, hemameba level, neutrophil counts, ASA classification, and comorbidities (including arrhythmia, hypertension, diabetes, CHD, history of stroke, and digestive system disease) (Table 1). The table depicted that the preoperative MOCA scores were significantly different between all three groups (group $\mathrm{N}: 23.83 \pm 3.21$, group $\mathrm{M}: 20.7 \pm 2.92$, group $\mathrm{S}$ : $15.93 \pm 4.75$; group $\mathrm{N}$ vs. group $\mathrm{M}: P=0.002$, group $\mathrm{N}$ vs. group S: $P \leq 0.001$, group $M$ vs. group $S: P \leq 0.001$ ).

3.2. Intraoperative Blood Pressure. No significant difference in intraoperative blood pressure was observed between the three groups (Table 1). The L-SBP in group S $(90.00 \mathrm{mmHg}$; IQR, 80.00-100.00) was numerically but not significantly lower than that in group $\mathrm{N}(100.00 \mathrm{mmHg}$; IQR, 83.75$102.75)$ and group $\mathrm{M}(100.00 \mathrm{mmHg}$; IQR, 90.00-113.75; $P$ $=0.083)$. The M-SBP and M-DBP showed a tendency to increase in group N (M-SBP: 20.00\%; IQR, 4.50-29.25. MDBP: 22.50\%; IQR, 13.00-35.00), group M (M-SBP: 17.00\%; IQR, 7.00-26.00. M-DBP: 23.00\%; IQR, 9.2528.50), and group S (M-SBP: 24.00\%; IQR, 13.00-29.00. MDBP: $27.00 \%$; IQR, 16.00-31.50), but no statistically significant differences were observed.
3.3. Postoperative Hemoglobin Level. The results shown in Table 1 indicate a significantly lower hemoglobin (T3) level in groups $\mathrm{M}(102.50 \pm 15.87 \mathrm{~g} / \mathrm{l})$ and $\mathrm{S}(102.58 \pm 9.11 \mathrm{~g} / \mathrm{l})$ than in group $\mathrm{N}(109.83 \pm 13.29 \mathrm{~g} / \mathrm{l})$ (group $\mathrm{N}$ vs. group $\mathrm{M}, P=0.022$; group $\mathrm{N}$ vs. group $\mathrm{S}, P=0.029$ ). However, hemoglobin levels measured at T2 did not follow this pattern.

3.4. Postoperative Inflammatory Indicators. In terms of postoperative inflammatory indicators (Table 1), CRP levels in group $\mathrm{N}(44.55 \pm 23.52 \mathrm{mg} / \mathrm{l})$ at $\mathrm{T} 3$ were significantly reduced compared to those in group $\mathrm{M}(62.35 \pm 32.31 \mathrm{mg} /$ 1) and group $S(61.13 \pm 30.24 \mathrm{mg} / \mathrm{l}) \quad(P=0.019$ and $P=$ 0.039 , respectively). The ESR showed a similar trend to CRP levels, but the difference was not significant. Additionally, no significant correlation was observed for leukocyte and neutrophil counts.

3.5. Postoperative Pain. In the follow-up of 103 patients at T2, 30 elderly patients experienced mild pain (group $\mathrm{N}, n$ $=14$ (46.7\%); group $\mathrm{M}, n=10$ (33.3\%); group $\mathrm{S}, n=6$ $(20.0 \%)), 53$ elderly patients experienced moderate pain (group N, $n=14$ (26.4\%); group $\mathrm{M}, n=24$ (45.3\%); group S, $n=15(28.3 \%))$, and 20 elderly patients experienced severe pain (group $\mathrm{N}, n=2(10 \%)$; group $\mathrm{M}, n=6(30 \%)$; group $\mathrm{S}$, $n=12(60 \%))$. As shown in Table 1 , a significant positive correlation was observed between the level of postoperative pain and POCD $\left(\chi^{2}=13.48, P=0.009\right)$, whereas a significant difference in the degree of pain at T3 was not evident.

3.6. Surgical Information. No statistically significant differences in surgery duration were observed among groups. Regarding the use of tourniquets (Table 1), among the 19 patients who used tourniquets throughout the course, 3 (15.8\%) had normal cognitive function, 9 (47.4\%) had mild POCD, and 7 (36.8\%) had severe POCD, but none of the differences were statistically significant $(P=0.363)$. In this study, among the 23 patients who required a second surgery, $4(17.4 \%)$ had normal cognitive function, $11(47.8 \%)$ had mild POCD, and $8(34.8 \%)$ had severe POCD. Although the probability of POCD was higher during the second operation, the difference was not significant $(P=0.352)$.

3.7. Multivariate Regression Analysis. In the multivariate regression analysis (Table 2), the preoperative MOCA score (OR: $0.632, P \leq 0.001$ ), postoperative CRP level (OR: 1.025, $P=0.038$ ), and postoperative pain at T2 (OR: 3.111, $P=$ $0.023)$ were independent risk factors for mild POCD. Meanwhile, with the exception of postoperative CRP levels (OR: $1.028, P=0.052$ ), the preoperative MOCA score (OR: $0.396, P \leq 0.001)$ and postoperative pain at T2 (OR: 9.348, $P=0.001)$ were also independent risk factors for severe POCD.

\section{Discussion}

After a retrospective study of 103 patients who underwent TKA, we found that preoperative MOCA scores largely determined the postoperative cognitive functional level. Severe postoperative decreases in hemoglobin levels, 
TABLe 1: Risk factor analyses of POCD.

\begin{tabular}{|c|c|c|c|c|}
\hline & $\begin{array}{l}\text { Group } \mathrm{N} \\
(n=30)\end{array}$ & $\begin{array}{l}\text { Group M } \\
(n=40)\end{array}$ & $\begin{array}{l}\text { Group S } \\
(n=33)\end{array}$ & $P$ value \\
\hline \multicolumn{5}{|l|}{ Part 1: patients' baseline data } \\
\hline Female, $n(\%)$ & $18(25.8)$ & $26(37.1)$ & $26(37.1)$ & 0.245 \\
\hline Age (year) & $66.97 \pm 6.33$ & $67.20 \pm 5.71$ & $67.18 \pm 5.97$ & 0.970 \\
\hline BMI $\left(\mathrm{kg} / \mathrm{m}^{2}\right)$ & $25.71 \pm 2.51$ & $27.07 \pm 3.90$ & $26.54 \pm 4.56$ & 0.264 \\
\hline SBP (mmHg) & $135.70 \pm 22.31$ & $140.15 \pm 15.76$ & $141.36 \pm 12.19$ & 0.404 \\
\hline DBP (mmHg) & $86.87 \pm 18.01$ & $83.63 \pm 7.49$ & $83.76 \pm 9.35$ & 0.928 \\
\hline Hemoglobin (g/l) & $133.03 \pm 14.81$ & $128.15 \pm 14.78$ & $130.12 \pm 11.29$ & 0.429 \\
\hline MoCA score & $23.83 \pm 3.21^{\mathrm{ab}}$ & $20.7 \pm 2.92^{\mathrm{ac}}$ & $15.93 \pm 4.75^{\mathrm{bc}}$ & $\leq 0.001$ \\
\hline $\mathrm{ESR}, \mathrm{mm} / \mathrm{h}$ (range) & $11.00(7.75,14.50)$ & $12.50(9.00,16.75)$ & $14.00(9.50,17.00)$ & 0.326 \\
\hline CRP, mg/l (range) & $2.10(1.47,4.37)$ & $2.65(1.32,4.55)$ & $3.00(1.95,4.20)$ & 0.701 \\
\hline Hemameba $\left(* 10^{9} / \mathrm{l}\right)$ & $5.50 \pm 1.25$ & $5.45 \pm 1.31$ & $5.52 \pm 1.25$ & 0.806 \\
\hline Neutrophils $\left(* 10^{9} / 1\right)$ & $3.07 \pm 0.74$ & $2.98 \pm 1.01$ & $2.88 \pm 0.94$ & 0.487 \\
\hline Arrhythmia, $n(\%)$ & $1(16.7)$ & $3(50)$ & $2(33.3)$ & 0.795 \\
\hline Hypertension, $n(\%)$ & $15(36.6)$ & $14(34.1)$ & $12(29.3)$ & 0.409 \\
\hline Diabetes, $n(\%)$ & $3(27.3)$ & $5(45.5)$ & $3(27.3)$ & 0.924 \\
\hline $\mathrm{CHD}, n(\%)$ & $2(33.3)$ & $3(50.0)$ & $1(16.7)$ & 0.765 \\
\hline Stroke, $n(\%)$ & $1(12.5)$ & $3(37.5)$ & $4(50.0)$ & 0.479 \\
\hline Digestive system disease, $n(\%)$ & $2(33.3)$ & $3(50.0)$ & $1(16.7)$ & 0.765 \\
\hline ASAI, $n(\%)$ & $14(27.5)$ & $20(39.2)$ & $17(33.3)$ & \\
\hline II, $n(\%)$ & $13(30.2)$ & $17(39.5)$ & $13(30.2)$ & 0.994 \\
\hline III, $n(\%)$ & $3(33.3)$ & $3(33.3)$ & $3(33.3)$ & \\
\hline \multicolumn{5}{|l|}{ Part 2: intraoperative blood pressure } \\
\hline Intraoperative SBP, mmHg (range) & $110.00(100.00,120.00)$ & $110.00(100.00,130.00)$ & $110.00(100.00,120.00)$ & 0.244 \\
\hline Intraoperative $\mathrm{DBP}, \mathrm{mmHg}$ (range) & $65.00(60.00,71.25)$ & $65.00(60.00,77.50)$ & $65.00(60.00,70.00)$ & 0.444 \\
\hline L-SBP, mmHg (range) & $100.00(83.75,102.75)$ & $100.00(90.00,113.75)$ & $90.00(80.00,100.00)$ & 0.083 \\
\hline M-SBP, \% (range) & $20.00(4.50,29.25)$ & $17.00(7.00,26.00)$ & $24.00(13.00,29.00)$ & 0.169 \\
\hline M-DBP, \% (range) & $22.50(13.00,35.00)$ & $23.00(9.25,28.50)$ & $27.00(16.00,31.50)$ & 0.381 \\
\hline \multicolumn{5}{|l|}{ Part 3: hemoglobin } \\
\hline Hemoglobin (T2) (g/l) & $114.90 \pm 14.16$ & $112.05 \pm 16.80$ & $113.79 \pm 11.68$ & 0.528 \\
\hline Hemoglobin (T3) (g/l) & $109.83 \pm 13.29^{\mathrm{de}}$ & $102.50 \pm 15.87^{\mathrm{d}}$ & $102.58 \pm 9.11^{\mathrm{e}}$ & 0.039 \\
\hline \multicolumn{5}{|l|}{ Part 4: inflammatory indicators } \\
\hline Postoperative ESR (mm/h) & $36.02 \pm 12.811$ & $45.71 \pm 19.50$ & $44.18 \pm 21.84$ & 0.066 \\
\hline Postoperative CRP (mg/l) & $44.55 \pm 23.52^{\mathrm{fg}}$ & $62.35 \pm 32.31^{\mathrm{f}}$ & $61.13 \pm 30.24^{\mathrm{g}}$ & 0.041 \\
\hline Leukocytes, $* 10^{9} / 1(\mathrm{~T} 2)$ & $9.34(7.03,11.34)$ & $8.62(7.74,11,00)$ & $8.99(7.64,11.16)$ & 0.941 \\
\hline Leukocytes, $* 10^{9} / 1$ (T3) & $6.14(5.40,7.45)$ & $6.15(5.34,7.36)$ & $6.63(5.22,7.40)$ & 0.724 \\
\hline Neutrophils, $* 10^{9} / 1(\mathrm{~T} 2)$ & $6.54(5.31,9.13)$ & $6.77(5.31,9.23)$ & $7.32(6.00,9.20)$ & 0.772 \\
\hline Neutrophils, $* 10^{9} / 1$ (T3) & $4.46(3.52,5.51)$ & $4.50(3.81,5.51)$ & $4.45(3.23,5.78)$ & 0.877 \\
\hline \multicolumn{5}{|l|}{ Part 5: postoperative pain } \\
\hline \multicolumn{5}{|l|}{ Pain (T2) } \\
\hline Mild, $n(\%)$ & $14(46.7)$ & $10(33.3)$ & $6(20.0)$ & \\
\hline Moderate, $n(\%)$ & $14(26.4)$ & $24(45.3)$ & $15(28.3)$ & $0.009^{*}$ \\
\hline Severe, $n(\%)$ & $2(10.0)$ & $6(30.0)$ & $12(60.0)$ & \\
\hline \multicolumn{5}{|l|}{ Pain (T3) } \\
\hline Mild, $n(\%)$ & $27(31.4)$ & $35(40.7)$ & $24(27.9)$ & \\
\hline Moderate, $n(\%)$ & $3(18.8)$ & $4(25.0)$ & $9(56.3)$ & 0.160 \\
\hline Severe, $n(\%)$ & $0(0.0)$ & $1(100.0)$ & $0(0.0)$ & \\
\hline Part 6: surgical information & & & & \\
\hline
\end{tabular}


TABLE 1: Continued.

\begin{tabular}{llll}
\hline & $\begin{array}{l}\text { Group N } \\
(n=30)\end{array}$ & $\begin{array}{l}\text { Group M } \\
(n=40)\end{array}$ & $\begin{array}{l}\text { Group S } \\
(n=33)\end{array}$ \\
\hline $\begin{array}{l}\text { Surgery duration, min } \\
\text { Tourniquets }\end{array} \quad$ value \\
$\quad$ Short time, $n(\%)$ & $27.30 \pm 19.005$ & $55.95 \pm 13.832$ & $61.18 \pm 16.918$ \\
$\quad$ Long time, $n(\%)$ & $3(15.8)$ & $31(36.9)$ & $26(31.0)$ \\
Second surgery & $26(32.5)$ & $9(47.4)$ & $7(36.8)$ \\
$\quad$ No, $n(\%)$ & $4(17.4)$ & $29(36.3)$ & $25(31.3)$ \\
$\quad$ Yes, $n(\%)$ & $11(47.8)$ & $8(34.8)$ \\
\hline
\end{tabular}

Values are means \pm SDs, numbers (\%), or medians and ranges. Abbreviations: CRP: C-reactive protein; CHD: coronary atherosclerotic heart disease; SBP: systolic blood pressure; DBP: diastolic blood pressure; ESR: erythrocyte sedimentation rate; BMI: body mass index; ASA, American Society of Anesthesiologists; SBP: systolic blood pressure; DBP: diastolic blood pressure; L-SBP: lowest systolic blood pressure; M-SBP: magnitude of blood pressure reduction in systolic blood pressure; M-DBP: magnitude of blood pressure reduction in diastolic blood pressure. ${ }^{\mathrm{a}} \mathrm{Group} \mathrm{N}$ vs. group $\mathrm{M}, P=0.002 .{ }^{\mathrm{b}} \mathrm{Group}$ $\mathrm{N}$ vs. group $\mathrm{S}, P \leq 0.001$. ${ }^{\mathrm{c}} \mathrm{Group} \mathrm{M}$ vs. group $\mathrm{S}, P \leq 0.001$. ${ }^{\mathrm{d}} \mathrm{Group} \mathrm{N}$ vs. group $\mathrm{M}, P=0.022$. ${ }^{\mathrm{e}}$ Group $\mathrm{N}$ vs. group $\mathrm{S}, P=0.029 .{ }^{\mathrm{f}} \mathrm{Group} \mathrm{N}$ vs. group $\mathrm{M}, P=$ 0.019. ${ }^{\mathrm{g}}$ Group N vs. group $\mathrm{S}, P=0.039 .{ }^{*} \chi^{2}=13.48, P=0.009$.

TABLE 2: Multivariate stepwise logistical regression analysis.

\begin{tabular}{|c|c|c|c|c|c|c|}
\hline & & OR & Corrected OR & $P$ value & Lower limit & Upper limit \\
\hline \multirow{4}{*}{ Mild POCD } & Preoperative MOCA & 0.702 & 0.632 & $\leq 0.001$ & 0.497 & 0.803 \\
\hline & Postoperative CRP & 1.024 & 1.025 & 0.038 & 1.001 & 1.049 \\
\hline & Hemoglobin (T3) & 0.959 & 0.957 & 0.094 & 0.909 & 1.008 \\
\hline & Pain (T2) & 2.042 & 3.111 & 0.023 & 1.169 & 8.280 \\
\hline \multirow{4}{*}{ Severe POCD } & Preoperative MOCA & 0.462 & 0.396 & $\leq 0.001$ & 0.281 & 0.559 \\
\hline & Postoperative CRP & 1.023 & 1.028 & 0.052 & 1.000 & 1.058 \\
\hline & Hemoglobin (T3) & 0.959 & 0.941 & 0.056 & 0.885 & 1.002 \\
\hline & Pain (T2) & 3.845 & 9.348 & 0.001 & 2.539 & 3.442 \\
\hline
\end{tabular}

Abbreviation: POCD: postoperative cognitive dysfunction.

increased levels of an inflammatory factor (CRP), and severe pain were associated with the occurrence of POCD. The increase in CRP levels was an independent risk factor for mild POCD, while the preoperative MOCA score and postoperative severe pain were independent risk factors for mild and severe POCD. However, intraoperative blood pressure, surgery duration, tourniquet usage pattern, and times of operation had no significant correlation with POCD.

Aging was associated with global declines in network segregation, integration, and module distinctiveness, and specific declines in distinctiveness of higher-order cognitive networks; this functional network deterioration was associated with poorer cognitive performance [11]. Understandably, the elderly are at a high risk of POCD [12]. However, we did not observe a statistically significant difference in age between the three groups. This apparent difference may be based on the comparison of middle-aged and elderly groups, rather than older groups. Another potential explanation is that patients with knee osteoarthritis who need TKA are relatively healthy (no serious systemic diseases), and even a few elderly patients were $>80$ years old.

Although an overall decline in the cognitive function level of elderly patients has been documented, individual differences are still significant, as represented by the preopera- tive MOCA scores which range from 8 to 30 in our study. We evaluated the basic cognitive functional status of patients using the preoperative MOCA to reduce the interference caused by individual differences. The preoperative MOCA score was an independent predictor of mild and severe POCD in our study. This finding is consistent with recent pooled evidence on the risk factors for POCD [13]. Ghaffary et al. also found a strong relationship between the patients' basic cognition and POCD after cardiac surgery [14]. A wise approach is to screen high-risk groups for POCD through the preoperative MOCA assessment in the clinic.

Combined with the characteristics of TKA surgery (e.g., pain, blood loss), we used the controlled hypotension technology on the one hand to reduce intraoperative bleeding and on the other hand to reduce the use of a tourniquet to alleviate the pain [15]. Cerebral hypoperfusion occurring with or as a result of controlled hypotension seems inevitable. Surprisingly, no international recommendations or high quality evidence for diagnostics and treatment of neurocognitive impairment that may arise from hypotension-related hypoperfusion is available $[16,17]$. Consistent with this result, this study reported the reduction in L-SBP, M-SBP, and M-DBP in patients with severe POCD, but the differences were not statistically significant. This lack of difference 
may be due to the existence of protective mechanisms in the brain, such as brain autoregulation function, cerebral blood flow reserve, and any factors (notably changes in arterial $\mathrm{CO}_{2}$ pressure) that decrease or increase cerebral blood flow $(\mathrm{CBF})$ [18]. A reasonable assumption is that controlled hypotension under general anesthesia is safe, but a risk of cerebral ischemia still exists for patients with prolonged severe hypotension during surgery.

The amount of blood loss during TKA is high, and different degrees of blood oozing will occur after TKA, which lead to further increases in blood loss. Severe intraoperative and postoperative blood loss caused by TKA has always been a concern [19]. The estimation of hemoglobin loss showed a significantly better estimation of blood loss than blood volume loss formulae [20]. Blood loss, including postoperative oozing, as reflected by reduced hemoglobin levels, might render a patient susceptible to a higher risk of POCD in the present study. The conclusion that the occurrence of cognitive dysfunction is affected by intraoperative blood loss in elderly patients has consistently been confirmed [21,22]. A deficiency in oxygen supply to the brain may result in cognitive dysfunction, which may explain the association between low hemoglobin levels and POCD [23]. A natural speculation is that transient anemia after TKA is also related to POCD. However, the authors evaluated 15,105 participants from the ELSA-Brazil Cohort Baseline and found no association between anemia and cognitive performance [24]. This difference may be caused by the use of different definitions for anemia and POCD, age of the participants, and other factors.

The general consensus is that the mechanism of postoperative cognitive dysfunction includes an inflammatory response $[25,26]$. Peripheral immune cells play a role in the immune inflammatory response in the hippocampus following surgery and the inhalation of anesthesia, and the inflammatory specific biomarkers associated with the destruction of the blood-brain barrier (BBB) [27]. Chronic inflammatory disease states produced by the genetic and environmental factors also increase the risk of endothelial dysfunction with increased BBB permeability [28]. In molecular biology, microglia are important cells that maintain the balance of inflammation in the brain [29]. The increased levels of peripheral inflammatory factors induced by surgical stimuli cause microglial activation [30], which produce cytokines and result in the production of a range of inflammatory factors in areas of the central nervous system (CNS), in particular but not restricted to the hippocampus [31]. Interestingly, neuroinflammation is considered as a doubleedged sword that exerts both detrimental and beneficial effects on the neurons. Inflammation-mediated neurotoxicity occurs as a consequence of microglial dysregulation and overactivation [32], whereas some data support that microglia stimulate myelin repair, remove toxic proteins from the CNS, and prevent neurodegeneration in individuals with chronic brain diseases [33, 34]. Microglial switching requires fine regulation, and more in-depth investigations are urgently needed [35]. Our study revealed that an elevated postoperative CRP level was an independent risk factor for mild POCD, but not severe POCD, which may be related to the dual effect of the inflammatory response.
In addition, the result may be different when the sample size is further increased.

Postoperative pain also affected POCD in elderly patients after TKA, which was more intuitive compared with the hemoglobin level and inflammatory response. Based on clinical research using the visual analogue scale (VAS) to determine the degree of postoperative pain, POCD correlated with postoperative pain in patients who underwent abdominal surgery using different surgical methods [36]. Furthermore, untreated pain might be an important etiological factor for POCD, and it is common in elderly patients following surgery for hip fracture [37]. The mechanisms by which pain (acute and chronic pain) induces cognitive dysfunction are complex. Acute pain may aggravate postoperative cognitive dysfunction (such as memory deficits) via neurotransmitters (increased dopamine (DA) levels in the cortex and decreased acetylcholine (ACH) levels in the hippocampus) and by changing the levels of inflammatory factors [38]. The administration of effective analgesics to elderly patients might have benefits in preventing cognitive decline in some domains [39]. Compared with the effects of intravenous analgesia, the postoperative epidural analgesia decreased the systemic inflammatory response, the perceived pain, and the incidence of POCD [40, 41]. Preemptive analgesia induced by a continuous femoral nerve block may promote the recovery of early cognitive function in elderly patients following TKA [42]. The effect on the level of cognitive function is one of the indicators of choice for analgesia.

Prolonged surgery duration may lead to increased blood loss and inflammatory responses, as well as increased use of anesthetic drugs. As shown in numerous studies, the duration of the operation affects the occurrence of POCD [43]. However, no correlation between the time of operation and the occurrence of POCD was observed in our study. This result is also consistent with some other studies [44]. This finding may be due to the fact that our patients were operated on by the same surgical team, and the duration of the surgery itself did not vary substantially.

Tourniquet use has been linked to adverse outcomes resulting from intraoperative and postoperative ischemia, such as nerve paralysis, soft-tissue damage, and thromboembolism [45]. Tourniquet-related ischemia-reperfusion injury during TKA leads to altered muscle protein metabolism, endothelial dysfunction, oxidative stress, and an inflammatory response [46]. Based on advantages of the use of a tourniquet for short periods in all aspects [47], we hypothesized that the short-term use of tourniquets also promoted cognitive recovery. This research indicates that the incidence of POCD in elderly patients with full tourniquet use was higher than that in the short-term tourniquet group, but the difference was not statistically significant. Some studies also found that the patients who experienced controlled hypotension but without tourniquet use during the operation had higher MOCA scores than those patients who used tourniquets [15]. Therefore, a reduced time of tourniquet use is recommended.

Secondary surgery is quite common in elderly patients with osteoarthritis of the knee. The result illustrated that 
the elderly patients are more likely to develop POCD after the second surgery, but the difference was not significant. A few studies examined the relationship between a second TKA and POCD. Future studies could expand the sample size to verify these finding and to further explore the most appropriate time interval between the two operations.

Combined with surgical characteristics, we comprehensively analyzed the risk factors for POCD in elderly patients after TKA, such as tourniquet use and secondary surgery, which were not included in previous studies. The effect of secondary surgery and tourniquet use on POCD needs to be confirmed by more large-sample studies. In the clinical work about knee replacement surgery, preoperative cognitive functional level assessment maybe screen high-risk groups for POCD, and it is reasonable to reduce the occurrence of POCD by reducing inflammatory response, controlling bleeding, and reducing postoperative pain.

\section{Conclusions}

Elderly patients with TKA often suffer from different levels of POCD. In the multivariate regression analysis, the preoperative cognitive function level, increased postoperative CRP level, and severe pain were independent risk factors for mild POCD. Moreover, the preoperative cognitive function level and severe pain contributed to POCD to a greater extent than the postoperative CRP level. The postoperative hemoglobin level may be related to the occurrence of POCD. These risk factors can be used to estimate the high-risk population and severity of POCD to guide clinical practice.

\section{Data Availability}

All the data involved in this study have been included within the article.

\section{Conflicts of Interest}

The authors have no conflicts of interest regarding the publication of this article to declare.

\section{Acknowledgments}

This work was supported by a grant from the Weifang Science and Technology Bureau (project number 2020YX009).

\section{References}

[1] K. Van Sinderen, L. A. Schwarte, and P. Schober, "Diagnostic criteria of postoperative cognitive dysfunction: a focused systematic review," Anesthesiology Research and Practice, vol. 2020, Article ID 7384394, 13 pages, 2020.

[2] D. L. McDonagh, J. P. Mathew, W. D. White et al., "Cognitive function after major noncardiac surgery, apolipoprotein E4 genotype, and biomarkers of brain injury," The Journal of the American Society of Anesthesiologists, vol. 112, no. 4, pp. 852-859, 2010.

[3] Z. S. Nasreddine, N. A. Phillips, V. Bédirian et al., "The Montreal cognitive assessment, MoCA: a brief screening tool for mild cognitive impairment," Journal of the American Geriatrics Society, vol. 53, no. 4, pp. 695-699, 2005.

[4] I. Rundshagen, "Postoperative cognitive dysfunction," Deutsches Ärzteblatt International, vol. 111, no. 8, pp. 119125, 2014.

[5] A. J. Claes, S. de Backer, P. Van de Heyning, A. Gilles, V. Van Rompaey, and G. Mertens, "Postoperative cognitive dysfunction after cochlear implantation," European Archives of OtoRhino-Laryngology, vol. 275, no. 6, pp. 1419-1427, 2018.

[6] N. Ciesielska, R. Sokołowski, E. Mazur, M. Podhorecka, A. Polak-Szabela, and K. Kędziora-Kornatowska, "Is the Montreal cognitive assessment (MoCA) test better suited than the mini-mental state examination (MMSE) in mild cognitive impairment (MCI) detection among people aged over 60 ? Meta-analysis," Psychiatria Polska, vol. 50, no. 5, pp. 10391052, 2016.

[7] J. Potocnik, K. Ovcar Stante, and M. Rakusa, "The validity of the Montreal cognitive assessment (MoCA) for the screening of vascular cognitive impairment after ischemic stroke," Acta Neurologica Belgica, vol. 120, no. 3, pp. 681-685, 2020.

[8] C. Tong, C. Huang, J. Wu, M. Xu, and H. Cao, “The prevalence and impact of undiagnosed mild cognitive impairment in elderly patients undergoing thoracic surgery: a prospective cohort study," Journal of Cardiothoracic and Vascular Anesthesia, vol. 34, no. 9, pp. 2413-2418, 2020.

[9] H. Delavaran, A. C. Jönsson, H. Lövkvist et al., "Cognitive function in stroke survivors: a 10-year follow-up study," Acta Neurologica Scandinavica, vol. 136, no. 3, pp. 187-194, 2017.

[10] A. J. Webb, S. T. Pendlebury, L. Li et al., "Validation of the Montreal cognitive assessment versus mini-mental state examination against hypertension and hypertensive arteriopathy after transient ischemic attack or minor stroke," Stroke, vol. 45, no. 11, pp. 3337-3342, 2014.

[11] J. S. X. Chong, K. K. Ng, J. Tandi et al., "Longitudinal changes in the cerebral cortex functional organization of healthy elderly," The Journal of Neuroscience, vol. 39, no. 28, pp. 5534-5550, 2019.

[12] Z. He, H. Cheng, H. Wu, G. Sun, and J. Yuan, "Risk factors for postoperative delirium in patients undergoing microvascular decompression," PLoS One, vol. 14, no. 4, article e0215374, 2019.

[13] L. A. Evered and B. S. Silbert, "Postoperative cognitive dysfunction and noncardiac surgery," Anesthesia and Analgesia, vol. 127, no. 2, pp. 496-505, 2018.

[14] S. Ghaffary, A. Hajhossein Talasaz, P. Ghaeli et al., "Association between perioperative parameters and cognitive impairment in post-cardiac surgery patients," The Journal of Tehran Heart Center, vol. 10, no. 2, pp. 85-92, 2015.

[15] J. Dong, S. Min, K. H. He, L. H. Peng, J. Cao, and W. Ran, "Effects of the nontourniquet combined with controlled hypotension technique on pain and long-term prognosis in elderly patients after total knee arthroplasty: a randomized controlled study," Journal of Anesthesia, vol. 33, no. 5, pp. 587-593, 2019.

[16] Ł. J. Krzych, M. P. Pluta, Z. Putowski, and M. Czok, "Investigating association between intraoperative hypotension and postoperative neurocognitive disorders in non-cardiac surgery: a comprehensive review," Journal of Clinical Medicine, vol. 9, no. 10, p. 3183, 2020.

[17] X. Feng, J. Hu, F. Hua, J. Zhang, L. Zhang, and G. Xu, “The correlation of intraoperative hypotension and postoperative cognitive impairment: a meta-analysis of randomized 
controlled trials," BMC Anesthesiology, vol. 20, no. 1, p. 193, 2020.

[18] J. C. Drummond, "Blood pressure and the brain: how low can you go?," Anesthesia and Analgesia, vol. 128, no. 4, pp. 759$771,2019$.

[19] W. J. Cundy, A. Theodoulou, C. M. Ling, J. Krishnan, and C. J. Wilson, "Blood loss in total knee arthroplasty," The Journal of Knee Surgery, vol. 30, no. 5, pp. 452-459, 2017.

[20] S. Jaramillo, M. Montane-Muntane, P. L. Gambus, D. Capitan, R. Navarro-Ripoll, and A. Blasi, "Perioperative blood loss: estimation of blood volume loss or haemoglobin mass loss?," Blood Transfusion, vol. 18, no. 1, pp. 20-29, 2020.

[21] J. Zhang, G. Liu, F. Zhang et al., "Analysis of postoperative cognitive dysfunction and influencing factors of dexmedetomidine anesthesia in elderly patients with colorectal cancer," Oncology Letters, vol. 18, no. 3, pp. 3058-3064, 2019.

[22] H. Zhou, F. Li, W. Ye et al., "Correlation between plasma circ RNA-089763 and postoperative cognitive dysfunction in elderly patients undergoing non-cardiac surgery," Frontiers in Behavioral Neuroscience, vol. 14, article 587715, p. 178, 2020.

[23] N. D. Croughwell, M. F. Newman, J. A. Blumenthal et al., "Jugular bulb saturation and cognitive dysfunction after cardiopulmonary bypass," The Annals of Thoracic Surgery, vol. 58, no. 6, pp. 1702-1708, 1994.

[24] J. B. R. Valladão Júnior, C. K. Suemoto, A. C. Goulart et al., "Anemia and cognitive performance in the ELSA-Brasil cohort baseline," The Journal of Neuropsychiatry and Clinical Neurosciences, vol. 32, no. 3, pp. 227-234, 2020.

[25] X. Liu, Y. Yu, and S. Zhu, "Inflammatory markers in postoperative delirium (POD) and cognitive dysfunction (POCD): a meta-analysis of observational studies," PLoS One, vol. 13, no. 12, article e0195659, 2018.

[26] L. Peng, L. Xu, and W. Ouyang, "Role of peripheral inflammatory markers in postoperative cognitive dysfunction (POCD): a meta-analysis," PLoS One, vol. 8, no. 11, article e79624, 2013.

[27] H. Zhu, W. Liu, and H. Fang, "Inflammation caused by peripheral immune cells across into injured mouse blood brain barrier can worsen postoperative cognitive dysfunction induced by isoflurane," BMC Cell Biology, vol. 19, no. 1, p. 23, 2018.

[28] B. Riedel, K. Browne, and B. Silbert, "Cerebral protection: inflammation, endothelial dysfunction, and postoperative cognitive dysfunction," Current Opinion in Anaesthesiology, vol. 27, no. 1, pp. 89-97, 2014.

[29] S. Saxena and M. Maze, "Impact on the brain of the inflammatory response to surgery," La Presse Médicale, vol. 47, no. 4, pp. e73-e81, 2018.

[30] I. C. Hoogland, C. Houbolt, D. J. van Westerloo, W. A. van Gool, and D. van de Beek, "Systemic inflammation and microglial activation: systematic review of animal experiments," Journal of Neuroinflammation, vol. 12, pp. 1-13, 2015.

[31] D. R. Skvarc, M. Berk, L. K. Byrne et al., "Post-operative cognitive dysfunction: an exploration of the inflammatory hypothesis and novel therapies," Neuroscience and Biobehavioral Reviews, vol. 84, pp. 116-133, 2018.

[32] M. L. Block, L. Zecca, and J. S. Hong, "Microglia-mediated neurotoxicity: uncovering the molecular mechanisms," Nature Reviews. Neuroscience, vol. 8, no. 1, pp. 57-69, 2007.

[33] I. Glezer, A. R. Simard, and S. Rivest, "Neuroprotective role of the innate immune system by microglia," Neuroscience, vol. 147, no. 4, pp. 867-883, 2007.
[34] A. R. Simard, D. Soulet, G. Gowing, J. P. Julien, and S. Rivest, "Bone marrow-derived microglia play a critical role in restricting senile plaque formation in Alzheimer's disease," Neuron, vol. 49, no. 4, pp. 489-502, 2006.

[35] Y. Tang and W. Le, "Differential roles of M1 and M2 microglia in neurodegenerative diseases," Molecular Neurobiology, vol. 53, no. 2, pp. 1181-1194, 2016.

[36] G. L. Gong, B. Liu, J. X. Wu, J. Y. Li, B. Q. Shu, and Z. J. You, "Postoperative cognitive dysfunction induced by different surgical methods and its risk factors," The American Surgeon, vol. 84, no. 9, pp. 1531-1537, 2018.

[37] P. M. Odor, I. Chis Ster, I. Wilkinson, and F. Sage, "Effect of admission fascia iliaca compartment blocks on postoperative abbreviated mental test scores in elderly fractured neck of femur patients: a retrospective cohort study," $B M C$ Anesthesiology, vol. 17, no. 1, pp. 1-8, 2017.

[38] X. Ding, X. Gao, Z. Wang et al., "Preoperative chronic and acute pain affects postoperative cognitive function mediated by neurotransmitters," Journal of Molecular Neuroscience, vol. 71, no. 3, pp. 515-526, 2021.

[39] G. Orhun, Z. Sungur, K. Koltka et al., "Comparison of epidural analgesia combined with general anesthesia and general anesthesia for postoperative cognitive dysfunction in elderly patients," Turkish Journal of Trauma and Emergency Surgery, vol. 26, no. 1, pp. 30-36, 2020.

[40] W. Yan, H. Mao, and P. Qiu, "Effects of different analgesia regimens on early post-operative cognitive dysfunction in elderly patients undergoing radical resection of cervical carcinoma," Experimental and Therapeutic Medicine, vol. 18, no. 2, pp. 1465-1469, 2019.

[41] G. Kristek, I. Radoš, D. Kristek et al., "Influence of postoperative analgesia on systemic inflammatory response and postoperative cognitive dysfunction after femoral fractures surgery: a randomized controlled trial," Regional Anesthesia and Pain Medicine, vol. 44, no. 1, pp. 59-68, 2019.

[42] L. Q. Deng, L. N. Hou, F. X. Song et al., "Effect of pre-emptive analgesia by continuous femoral nerve block on early postoperative cognitive function following total knee arthroplasty in elderly patients," Experimental and Therapeutic Medicine, vol. 13, no. 4, pp. 1592-1597, 2017.

[43] Y. Fan, X. Liu, S. Wu, and Y. Liu, "The risk factors for cognitive dysfunction in elderly patients after laparoscopic surgery: a retrospective analysis," Medicine, vol. 100, no. 2, article e23977, 2021.

[44] N. Kotekar, C. S. Kuruvilla, and V. Murthy, "Post-operative cognitive dysfunction in the elderly: a prospective clinical study," Indian Journal of Anaesthesia, vol. 58, no. 3, pp. 263268, 2014.

[45] T. O. Smith and C. B. Hing, "Is a tourniquet beneficial in total knee replacement surgery?: a meta-analysis and systematic review," The Knee, vol. 17, no. 2, pp. 141-147, 2010.

[46] P. Leurcharusmee, P. Sawaddiruk, Y. Punjasawadwong, N. Chattipakorn, and S. C. Chattipakorn, "The possible pathophysiological outcomes and mechanisms of tourniquetinduced ischemia-reperfusion injury during total knee arthroplasty," Oxidative Medicine and Cellular Longevity, vol. 2018, Article ID 8087598, 15 pages, 2018.

[47] L. E. Rasmussen, H. A. Holm, P. W. Kristensen, and P. Kjaersgaard-Andersen, "Tourniquet time in total knee arthroplasty,” The Knee, vol. 25, no. 2, pp. 306-313, 2018. 